High density experiments in TCV ohmically heated and L-mode plasmas

This content has been downloaded from IOPscience. Please scroll down to see the full text. 2015 Plasma Phys. Control. Fusion 57025002

(http://iopscience.iop.org/0741-3335/57/2/025002)

View the table of contents for this issue, or go to the journal homepage for more

Download details:

IP Address: 128.178.125.54

This content was downloaded on 19/12/2014 at 11:57

Please note that terms and conditions apply. 


\title{
High density experiments in TCV ohmically heated and L-mode plasmas
}

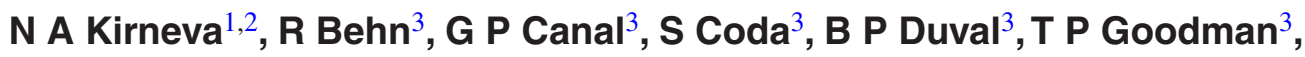 \\ B Labit ${ }^{3}$, N A Mustafin 1,2 , A N Karpushov ${ }^{3}$, A Pochelon ${ }^{3}$, L Porte ${ }^{3}$, \\ O Sauter ${ }^{3}$, M Silva ${ }^{3}$, B Tal ${ }^{4}$, V Vuille ${ }^{3}$ and the TCV Team \\ ${ }^{1}$ Institute of Physics of Tokamaks, National Research Center 'Kurchatov Institute', 123182 Kurchatov \\ Sq., 1, Moscow, Russia \\ 2 National Research Nuclear University MEPhI (Moscow Engineering Physics Institute), 115409, \\ Kashirskoe Sh., 31, Moscow, Russia \\ ${ }^{3}$ Ecole Polytechnique Fédérale de Lausanne (EPFL), Centre de Recherches en Physique des Plasmas, \\ CH-1015 Lausanne, Switzerland \\ ${ }^{4}$ Institute for Particle and Nuclear Physics, Wigner Research Centre for Physics, Hungarian Academy of \\ Sciences, Association EURATOM, PO Box 49 H-1525, Budapest, Hungary
}

E-mail: nkirneva@gmail.com

Received 30 May 2014, revised 10 October 2014

Accepted for publication 11 November 2014

Published 5 December 2014

\begin{abstract}
Recent experiments have been performed on the Tokamak à configuration variable (TCV) to investigate the confinement properties of high density plasmas and the mechanism behind the density limit. In a limiter configuration with plasma elongation $\kappa=1.3-1.4$ and triangularity $\delta=0.2-0.3$ the operational density range has been extended up to 0.65 of the Greenwald density at $I_{\mathrm{p}}=200 \mathrm{kA}\left(q_{95}=3.7\right)$ and even to the Greenwald value at low plasma current $I_{\mathrm{p}}=110 \mathrm{kA}\left(q_{95}=7\right)$. A transition from the linear to the saturated ohmic confinement regime is observed at high density $\sim 0.4 n_{\mathrm{GW}}$. A further density increase leads to sawtooth stabilization and is accompanied by a decrease of the energy and particle confinement times. The development of the disruption at the density limit was preceded by sawtooth stabilization. It is shown that electron cyclotron heating leads to the prevention of sawtooth stabilization and then to the increase of the density limit value.
\end{abstract}

Keywords: high density discharges, confinement, MHD activity, plasma current redistribution, density limit

(Some figures may appear in colour only in the online journal)

\section{Introduction}

A crucial recent event in the field of controlled nuclear fusion was the beginning of the construction of the 'International Thermonuclear Experimental Reactor'(ITER). One of the key issues to be addressed by this device is the demonstration of burning fusion plasma. To achieve its goals, the tokamakreactor has to operate close to the maximum design parameters, in particular the operational density is required to be close to the Greenwald density limit, $\bar{n}_{\mathrm{e}} \geq 0.85 n_{\mathrm{GW}}$ [1]. However some physics issues in such regime remain open and require additional experimental efforts in present day machines to prepare for efficient ITER operation.
The line-averaged density value ('Greenwald density') $n_{\mathrm{GW}}=I_{\mathrm{p}} /\left(\pi a^{2}\right)\left(\left(10^{20} \mathrm{~m}^{-3}\right)\right.$, where $I_{\mathrm{p}}$ is the plasma current (MA) and a the minor radius (m)) has been proposed in [2] as an empirical density limit, based on the analysis of a database including several tokamaks. It was later shown that the density limit value can be exceeded by the application of additional heating [3], by peaking of the current profile [4] and by modification of the density profile (see [5] and references therein). However the Greenwald density still remains a parameter that scales the critical densities for several processes in tokamak plasmas including the transition between confinement regimes [5].

In most cases, an increase in density results in an increase in effective collisionality, which scales as $n_{\mathrm{e}} / T_{\mathrm{e}}^{2}$. Several 
devices with dominant ion heating and mixed (ion + electron) heating demonstrated density peaking with collisionality decrease [6]. However there is some evidence of an opposite dependence for regimes with dominant electron heating, i.e. density flattening with collisionality decrease $[7,8]$. This variable peaking trend together with the issue of the dependence of the confinement properties on the plasma density appear to be among the critical issues for a future reactor, since the density profile determines the fusion yield and the bootstrap current fraction.

The development of disruption prevention algorithms seem also to be an important task for present day machines. The density limit disruption is usually considered to be the result of a tearing instability with poloidal wave number $m=2$ and toroidal wave number $n=1$ due to the cooling of the plasma periphery, followed by the growth of a $m / n=2 / 1$ island [1]. The model described in [9] (see also references therein) attributes the $m=2 / n=1$ island development to a decrease of the magnetic shear in the plasma core. In conventional regimes with $q(0) \sim 1$ this results in $m=1 / n=1$ mode growth and penetration to the outer region of $q=3 / 2,2$, causing a decrease of the magnetic shear in the vicinity of the $q=2$ surface, which destabilizes the $m=2 / n=1$ tearing mode. (In the case $q(0)>1$ the disruption development may be initiated by modes with higher numbers.) Impurity accumulation is considered as a possible mechanism behind the 'loss' of magnetic shear. In [10] the origin of the density limit disruption is explained in terms of radiative island development: the modification of the plasma current profile by an imbalance of heating and radiation inside the rational surface leads to the seed island generation. This picture is consistent with that presented in [9]. Additional heating in the island region or in the inner part of the plasma (that increases the heat flux through the island region) is usually considered as an effective mechanism of disruption prevention.

The Tokamak à configuration variable (TCV) is well suited for experiments aimed at studying the energy and particle confinement behavior in regimes with high density or at the density limit and for investigations of the effect of localized heating on the density limiting processes. This is a device capable of highly variable plasma configurations, equipped with a wide set of diagnostics including MHD diagnostics, multichannel soft x-ray (SXR) diagnostics and a multichannel bolometer system for the measurement of radiation losses; additionally, it is equipped with a very flexible electron cyclotron (EC) heating system, consisting of 6 gyrotrons of frequency $82.7 \mathrm{GHz}$ for the injection of power (0.5 MW per gyrotron) at the second harmonic (X2) of the EC resonance and 3 gyrotrons at $118 \mathrm{GHz}$, the third harmonic (X3). The beam launching geometry can be controlled for each X2 gyrotron and for the X3 set separately during a plasma discharge.

The material in this paper is structured as follows. The specific experimental conditions are described in section 2 , the value of the limit density in ohmically heated plasmas and its dependence on the plasma current are discussed in section 3, and observations of the high density regime and of the effect of EC heating $(\mathrm{ECH})$ are treated in section 4 . The modeling of

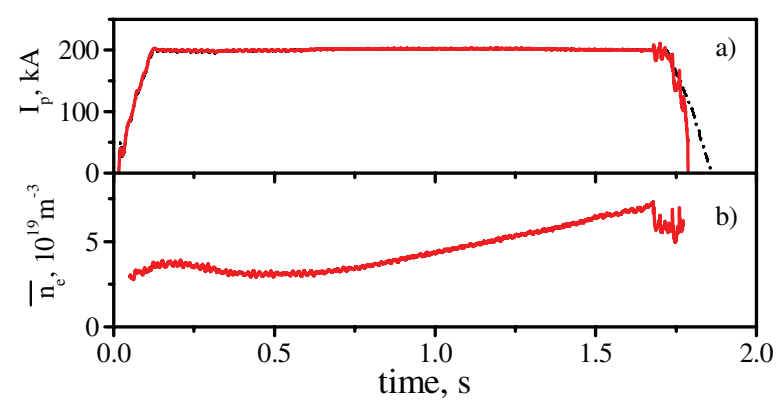

Figure 1. Typical scenario of high density experiments in TCV (shot 45181). Traces of plasma current $(a)$ and line averaged plasma density measured along the central chord $(b)$. The plasma current in a similar discharge without disruption is indicated by the dasheddotted line in $(a)$.

the current profile redistribution at high density and the effect of ECH on the current profile is presented in section 5. The confinement properties of TCV high density plasmas are presented in section 6 . The discussion of the experimental results and conclusions are given in section 7 .

\section{Experimental conditions}

These experiments were performed in plasmas with moderate elongation $\kappa=1.3-1.4$, positive triangularity $\delta_{95}=0.2-0.3$ at different plasma current values ranging from $I_{\mathrm{p}}=110 \mathrm{kA}$ $\left(q_{95}=7\right)$ to $I_{\mathrm{p}}=270 \mathrm{kA} \quad\left(q_{95}=2.9\right)$. The corresponding Greenwald density values are $n_{\mathrm{GW}}=5.1 \times 10^{19} \mathrm{~m}^{-3}$ for $I_{\mathrm{p}}=110 \mathrm{kA}$ and $n_{\mathrm{GW}}=1.1 \times 10^{20} \mathrm{~m}^{-3}$ for $I_{\mathrm{p}}=270 \mathrm{kA}$. Most of the experiments were in ohmically heated plasmas, however discharges with ECH were also developed to investigate the plasma response at high density and the possibility of disruption prevention.

In these experiments the line-averaged density was obtained from a far-infrared (FIR) interferometer, the electron density and temperature profiles were measured by Thomson scattering, and the ion temperature profile was provided by a charge-exchange recombination spectroscopy (CXRS). Plasma MHD activity was analyzed using the data from 4 poloidal arrays of magnetic probes, toroidally separated by $90^{\circ}$, and 2 toroidal arrays (of 16 equally spaced probes), and from the analysis of soft $x$-ray emission recorded by a multichord duplex multiwire proportional soft x-ray (DMPX) detector. The sawtooth dynamics were also investigated using the DMPX diagnostic. The sawtooth inversion radius was evaluated by Abel inversion of the DMPX data. Total radiation losses and their radial distribution were measured by multichannel Absolute Extreme UltraViolet Bolometer cameras (AXUV) and foil bolometer systems. The line-averaged density measured along the central chord was controlled with a feedback loop.

\section{The value of the limit density in ohmic discharges}

The typical experimental scenario for ohmic discharges is presented in figure 1. 

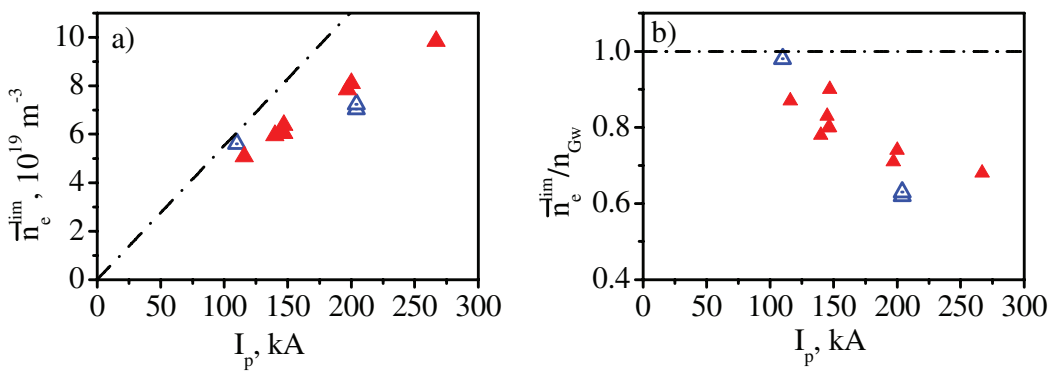

Figure 2. Dependencies on plasma current of the limit density value $(a)$ and of the ratio of the limit density to the Greenwald density $(b)$. Open triangles_-data from December 2011, closed symbols_-data from March 2013. The Greenwald density is shown by the dasheddotted line.
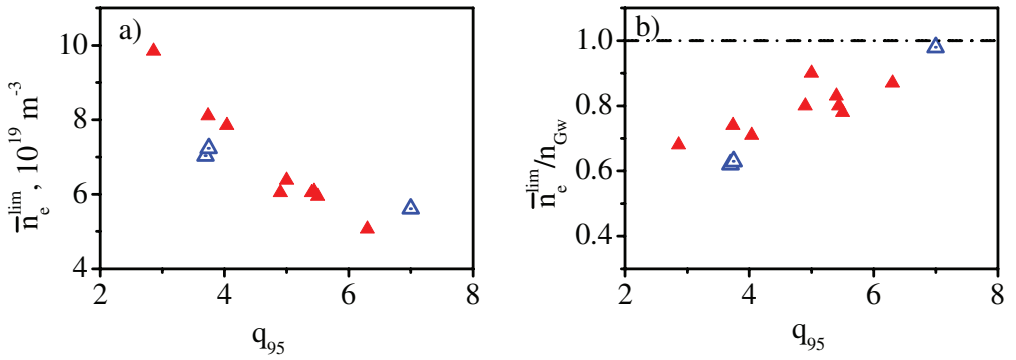

Figure 3. Dependence of the limit density value on $q_{95}$ absolute $(a)$ and normalized to the Greenwald density $(b)$. Open triangles—-data from December 2011, closed symbols—data from March 2013.

Table 1. Effective radius of the current channel and ratio $\bar{n}_{\mathrm{e}}^{\lim } / n_{\mathrm{GW}}^{\bmod }$ for different shots with different total plasma current.

\begin{tabular}{lllll}
\hline Shot No & Heating type & $I_{\mathrm{p}}(\mathrm{kA})$ & $a_{\mathrm{cur}} / a$ & $\bar{n}_{\mathrm{e}}^{\lim } / n_{\mathrm{GW}}^{\bmod }$ \\
\hline 46801 & $\mathrm{OH}$ & 270 & 0.88 & 0.53 \\
45180 & $\mathrm{OH}$ & 200 & 0.86 & 0.46 \\
46845 & $\mathrm{OH}$ & 145 & 0.82 & 0.53 \\
\hline
\end{tabular}

A maximal value of plasma limit density $\bar{n}_{\mathrm{e}}^{\lim }=9.85 \times 10^{19} \mathrm{~m}^{-3}=0.64 n_{\mathrm{GW}}$ was achieved in the regime with $I_{\mathrm{p}}=270 \mathrm{kA}$, whereas a value close to the Greenwald limit density was reached in the low current regime: $\bar{n}_{\mathrm{e}}=5.6 \times 10^{19} \mathrm{~m}^{-3}=n_{\mathrm{GW}}$. The dependence of the limit density value on plasma current is shown in figure 2 . The data from two distinct sets of experiments separated in time (December 2011 and March 2013) are depicted in the same plot. A similar dependence but in terms of $q_{95}$ is presented in figure 3. It is seen that the experiments are well reproducible for the trend and absolute values of the limit density. The value of the limit density increases with the plasma current but more slowly than predicted by the (linear) Greenwald formula.

A similar trend of the variation of $\bar{n}_{\mathrm{e}}^{\mathrm{lim}} / n_{\mathrm{GW}}$ with plasma current was obtained in the T-10 tokamak in discharges with additional EC heating [4]. It was shown that the replacement of the plasma minor radius by an effective radius of plasma current channel, $a_{\text {cur }}$, in the Greenwald formula restores a ratio $\bar{n}_{\mathrm{e}}^{\lim } / n_{\mathrm{GW}}=$ const $\sim 0.8$ in the whole plasma current range. The value of $a_{\text {cur }}$ was defined in [4] as the minor radius of the region where $95 \%$ of the total plasma current is concentrated. The value of $a_{\text {cur }}$ was estimated using the ASTRA code [11] for three representative TCV shots from the set shown in figure 2 and taken at different $I_{p}$ values (table 1). The electron temperature and density profiles were provided by Thomson
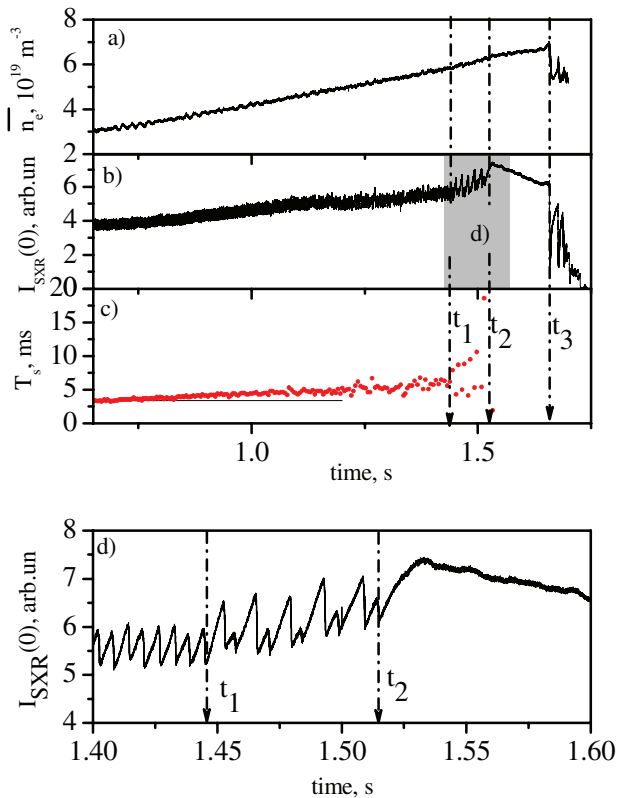

Figure 4. TCV shot 45180 . Traces of line averaged density $(a)$, SXR emission measured along a central chord $(b)$ and $(d)$ for a zoom-in, calculated value of the sawtooth period $(c)$. The times of the start of the sawtooth shape modification, of the start of the sawtooth-free phase and of the disruption are denoted as $t_{1}, t_{2}, t_{3}$, respectively.

scattering, while $Z_{\text {eff }}$ was assumed to be about 2 (derived from the loop voltage assuming neoclassical resistivity). The plasma conductivity and the bootstrap current were calculated from the neoclassical formula proposed in [12]. The calculated $a_{\text {cur }} / a$ and $\bar{n}_{\mathrm{e}}^{\lim } / n_{\mathrm{GW}}^{\bmod }$ values, where $n_{\mathrm{GW}}^{\bmod }=I /\left(\pi a_{\mathrm{cur}}^{2}\right)$, are shown in table 1 . The calculation indicates that the effective radius of the plasma current channel shrinks slightly 


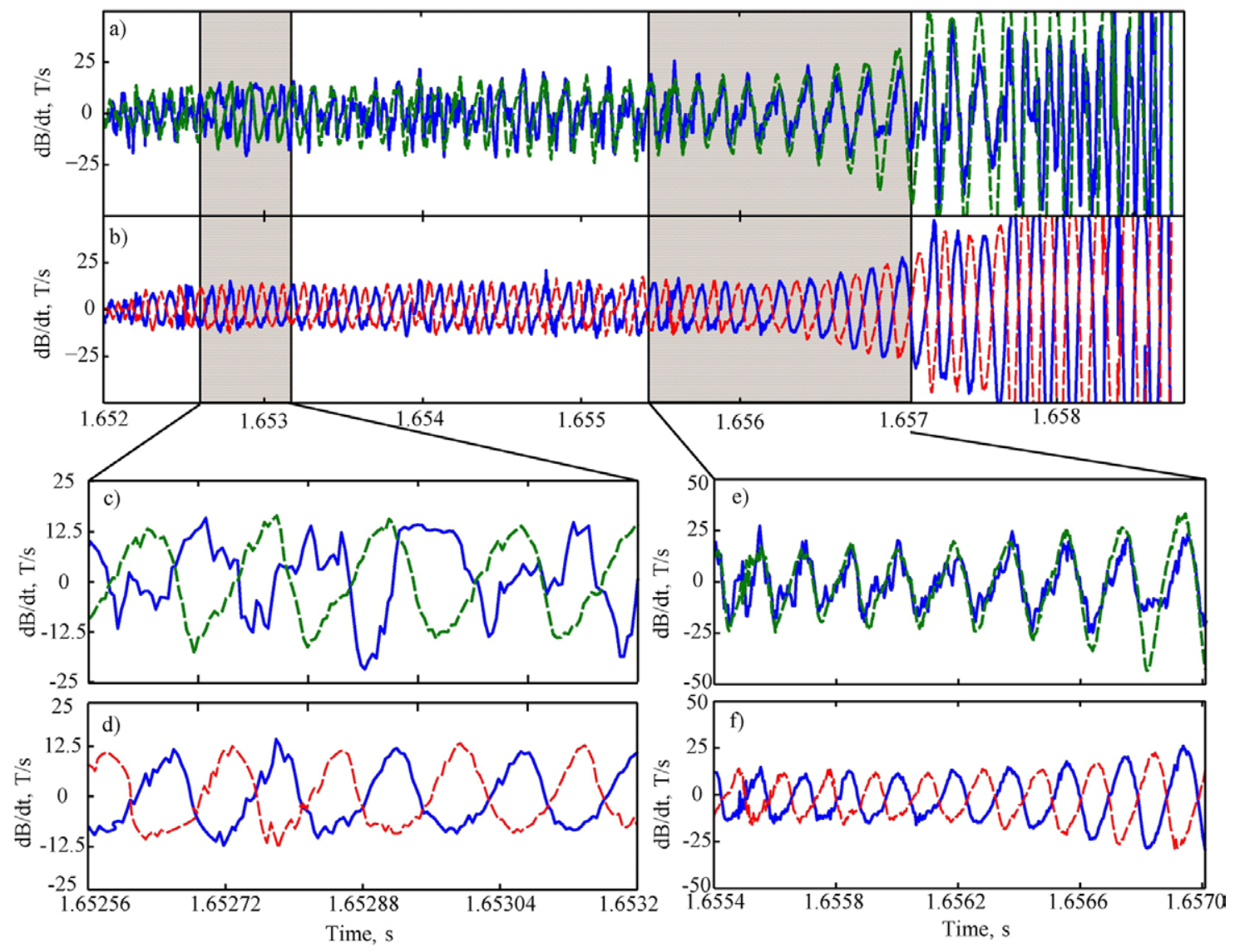

Figure 5. Shot 45180 (same as in figure 4). Illustration of MHD activity development close to the density limit (disruption time is $t_{\mathrm{dis}}=1.6585 \mathrm{~s}$ ): (a) traces from HFS (dashed curve) and LFS (solid curve) MHD probes on the plasma equatorial plane and at the same toroidal location; $(b)$ traces of two LFS MHD probes on the plasma equatorial plane and toroidally separated by $180^{\circ} ;(c),(d)$ and $(e),(f)$ show zooms of the same traces.

with a decrease in plasma current, reducing the scattering in $\bar{n}_{\mathrm{e}}^{\lim } / n_{\mathrm{GW}}^{\bmod }$ vs plasma current. Since the value of $n_{\mathrm{GW}}^{\bmod }$ is defined by $a_{\text {cur }}$ instead of the value of minor radius a, then it becomes higher than $n_{\mathrm{GW}}$ and the modified ratio $\bar{n}_{\mathrm{e}}^{\lim } / n_{\mathrm{GW}}^{\bmod }$ remains well below 1 .

\section{Properties of the high density regime}

\subsection{Ohmic discharges}

The increase in plasma density in these TCV experiments was accompanied by an increase in sawtooth period, a modification of the sawtooth shape and a reduction in their regularity, followed by the disappearance of sawtooth oscillations altogether (figure 4). In the regime with $I_{\mathrm{p}}=200 \mathrm{kA}\left(q_{95}=3.7\right)$ normal sawtooth oscillations with period $T_{\mathrm{s}}=5 \mathrm{~ms}$ are seen until the density reaches $\sim 80 \%$ of its limit value $\left(\bar{n}_{\mathrm{e}}^{\lim }\right)$, at which point the sawtooth shape changes (figures $4(b)$ and $(d)$ ): partial crashes appear and the sawtooth period becomes irregular (figure $4(c)$ ). The sawtooth period shown in figure $4(c)$ is calculated as the time interval between two adjacent internal crashes.

A sawtooth-free phase starts at $\left(\bar{n}_{\mathrm{e}}\right)_{\mathrm{SF}} \approx 0.9 n_{\text {lim }}$ and is followed by a giant crash at $\bar{n}_{\mathrm{e}}=\bar{n}_{\mathrm{e}}^{\lim }$. In most of the discharges MHD activity was not observed on either the MHD or SXR signals during the whole sawtooth free phase $(\sim 100-150 \mathrm{~ms})$ until a short interval just before the crash $(\sim 1-5 \mathrm{~ms})$. During this time interval an MHD perturbation becomes visible on different diagnostics. Figure 5 illustrates the evolution of signals from poloidally and toroidally separated MHD probes. Figures 5(a), $(c)$ and $(e)$ show traces of two poloidally separated magnetic probes: both are placed in the equatorial plane of the plasma column, one on the low-field side (LFS) and one on the high-field side (HFS). At the beginning of MHD mode development both signals are out of phase (see time interval $[1.652 ; 16535] \mathrm{s}$ in figure 5), indicating that the MHD mode has an odd poloidal mode number. Then, a second mode appears on the probe signals (time interval $[1.655,1.657] \mathrm{s}$ in figure 5), superposed on the first one and displaying an even poloidal mode number. Figures $5(b),(d)$ and $(f)$ show traces of two toroidally separated $\left(180^{\circ}\right)$ magnetic probes. The signals are out of phase, indicating an odd toroidal mode number for both modes. Basing on the set of data the first mode is identified as $m=1 / n=1$ and the second one as $m=2 / n=1$. The amplitudes of both modes grow until a crash occurs. This crash triggers again an $m=2 / n=1$ mode, developing similarly to that described in [13], causing a significant decrease of the plasma stored energy. This mode is stabilized within $\sim 1 \mathrm{~ms}$. The rest of the discharge is a sequence of sawtooth-like crashes (figure 4) with a decrease in plasma density, causing 

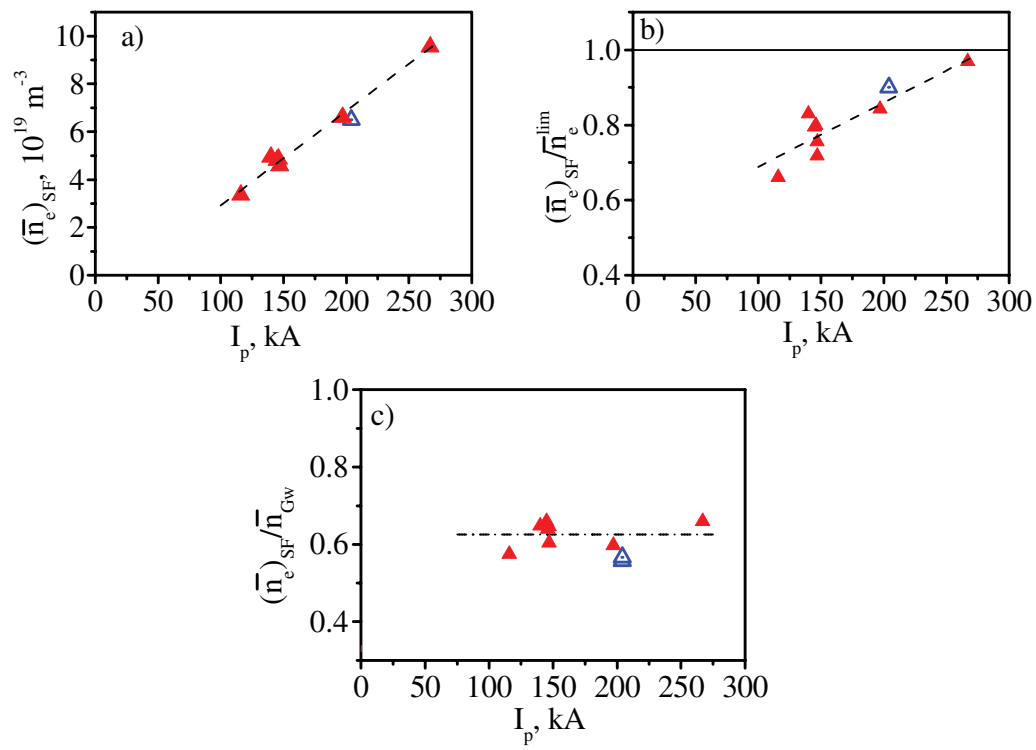

Figure 6. Dependence of the critical density value at the start of the sawtooth-free phase $(a)$ and of the ratio of this critical density to the limit one $(b)$ and to the Greenwald density $(c)$ on plasma current. Open triangles—data from December 2011 campaign, closed symbolsdata from March 2013

increased gas-puffing through feedback control and, as a consequence, additional plasma cooling until the discharge is terminated (about $50 \mathrm{~ms}$ after $\bar{n}_{\mathrm{e}}=\bar{n}_{\mathrm{e}}^{\lim }$ was reached).

The value of the critical density at which the sawtooth-free phase starts, $\left(\bar{n}_{\mathrm{e}}\right)_{S F}$, depends on the value of the plasma current (figure 6). The value of the difference between $\left(\bar{n}_{\mathrm{e}}\right)_{\mathrm{SF}}$ and $\bar{n}_{\mathrm{e}}^{\lim }$ is reduced at higher plasma current showing the trend of a shortening of the sawtooth-free phase with the increase of plasma current (figures $6(b)$ and 7 ). The ratio $\left(\bar{n}_{\mathrm{e}}\right)_{\mathrm{SF}} / n_{\mathrm{GW}}$ remains $\sim$ constant in the whole investigated range of plasma current values (figure 6(c)).

In discharges with lower plasma current $\left(I_{p}=145\right.$ and $115 \mathrm{kA}$ ) several internal crashes are observed during the sawtooth-free phase (figure 7). These crashes do not lead to a disruption, however they disturb the plasma column significantly, leading to increased radiation losses, decreased plasma energy content and perturbation of the plasma current (figure 8). The MHD mode structure prior to the crash is similar to the mode before the major disruption: the process starts with a more pronounced odd poloidal-number mode before the even mode becomes visible.

The modification of the sawtooth behavior correlates with an increase in density peaking. We choose to describe the density peaking by two parameters:

$$
F(q=1)=\frac{2 \times\left(n_{\mathrm{e}} L\right)_{7}}{\left(n_{\mathrm{e}} L\right)_{4}+\left(n_{\mathrm{e}} L\right)_{11}} \approx \frac{\left(n_{\mathrm{e}} L\right)_{0}}{\left(n_{\mathrm{e}} L\right)_{q=1}},
$$

and

$$
F(a / 2)=\frac{2 \times\left(n_{\mathrm{e}} L\right)_{7}}{\left(n_{\mathrm{e}} L\right)_{3}+\left(n_{\mathrm{e}} L\right)_{13}} \approx \frac{\left(n_{\mathrm{e}} L\right)_{0}}{\left(n_{\mathrm{e}} L\right)_{a / 2}},
$$

where $\left(n_{\mathrm{e}} L\right)_{i}$ is the line integrated density measured by the $i$ th vertical channel of the FIR interferometer. The following channels were used: channel 3 at $R=1.00 \mathrm{~m}$, channel 4 at $R=0.97 \mathrm{~m}$, channel 7 at $R=0.87 \mathrm{~m}$, channel 11 at $R=0.77 \mathrm{~m}$ and channel 13 at $R=0.72 \mathrm{~m}$. The radial magnetic axis position

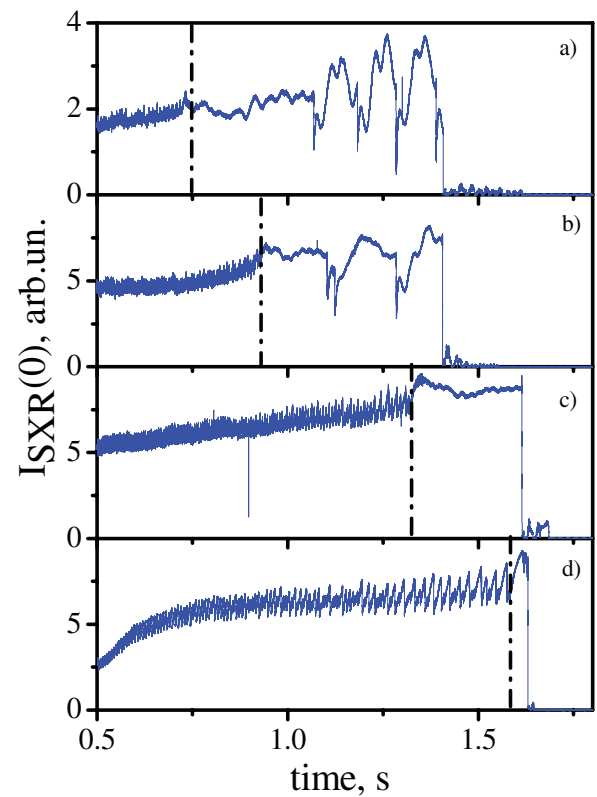

Figure 7. SXR emission measured along a central chord: $(a)$ shot $46803, I_{\mathrm{p}}=115 \mathrm{kA},(b)$ shot $46845, I_{\mathrm{p}}=145 \mathrm{kA},(c)$ shot 46796 , $I_{\mathrm{p}}=200 \mathrm{kA},(d)$ shot $46801, I_{\mathrm{p}}=270 \mathrm{kA}$. The start of the sawtoothfree phase is shown by a dashed-dotted line in each plot.

derived from the equilibrium reconstruction is $R=0.88 \mathrm{~m}$ at $t=1.3 \mathrm{~s}$ and moves outward very slightly (within $\sim 3 \mathrm{~mm}$ ) during the density ramp. Formula (1) describes the density peaking inside the $q=1$ zone, whereas formula (2) describes the density peaking inside the $a / 2$ region $(a=0.24 \mathrm{~m}$ being the plasma minor radius).

Figure 9 illustrates the modification of the density profile in the core region. Figure 10 demonstrates change in the radiative power distribution evaluated from multichannel bolometer measurements. The increase in line averaged density leads to an increase in density peaking in the center (figure $9(b)$ ). In accordance with the data presented in 


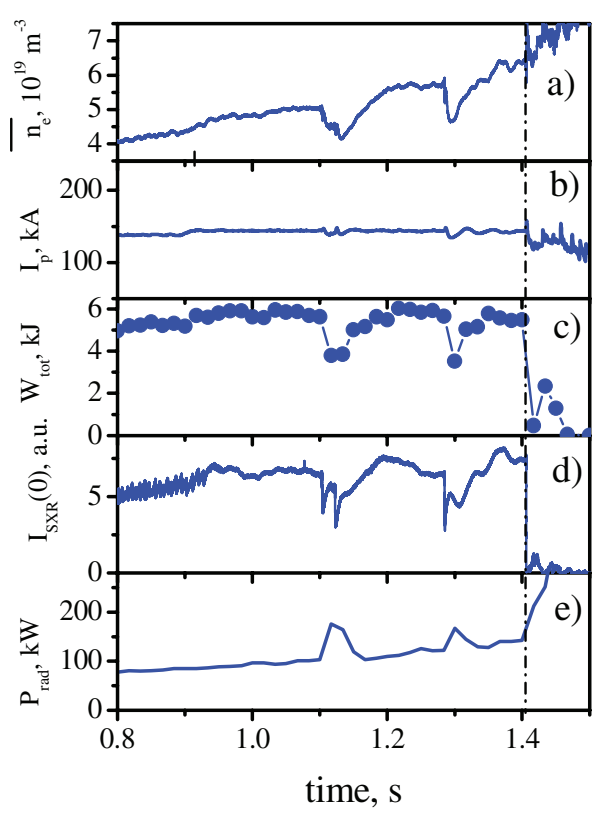

Figure 8. Traces of line averaged plasma density $(a)$, plasma current $(b)$, plasma energy content $(c)$, SXR emission measured along a central chord $(d)$ and total radiation losses $(e)$ for TCV shot $46845\left(I_{\mathrm{p}}=145 \mathrm{kA}\right)$. The disruption time is marked by a dasheddotted line.

figure $4(c)$, the sawtooth period also increases monotonically with density. At $t \sim t_{1}$, when the change in sawtooth shape becomes visible, a more global modification of the density profile occurs, as shown in figure $9(c)$. The density increase and the peaking of the density profile lead to an increase in core radiation (figure 10). The plasma current profile flattens due to the temperature profile flattening, causing the observed modification in sawtooth behavior and the increase in sawtooth period (figures $4(c)$ and $(d)$ ). In turn, the change in sawtooth behavior leads to an increase in both density and impurity peaking (figures $9(b)$ and $(c)$ and figure $10(b)$ and $(c)$ respectively), further amplifying the current redistribution and finally resulting in a sawtoothfree phase. The density peaking saturates at the beginning of the sawtooth-free phase.

The density peaking in the central region correlates with the increase of the sawtooth period, with a feedback process due both to the influence of the density peaking on the current profile redistribution and to the possible decrease of the density profile mixing (flattening) due to the increase in sawtooth period. Additionally, the effect of density peaking in the core during a density ramp can also be enhanced by a change in anomalous transport. For example, drift wave stability will change due to the change of the gradient lengths $L_{\mathrm{n}}=-\left(\left(\mathrm{d} n_{\mathrm{e}} / \mathrm{d} r\right) / n_{\mathrm{e}}\right)^{-1}, \quad L_{\mathrm{T}}=-\left(\left(\mathrm{d} T_{\mathrm{e}} / \mathrm{d} r\right) / T_{\mathrm{e}}\right)^{-1}$ and the increase in plasma collisionality. Therefore the balance between inward and outward particle fluxes governed by drift turbulence will change [8]; the relative role of the neoclassical particle flux may also increase and enhance the density peaking. Measurements of turbulence spectra and comparisons to numerical code predictions would be required to put these speculations on quantitative grounds.

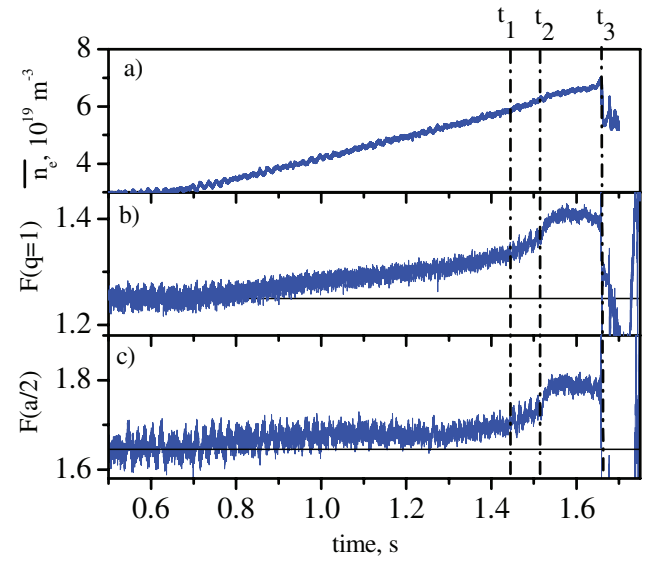

Figure 9. Density peaking: TCV shot 45180 -ohmic discharge with a density limit disruption. Line averaged plasma density measured along a central chord $(a)$, density peaking factor inside $q=1(b)$ and inside $a / 2(c)$. The times of change in sawteeth shape $\left(t_{1}\right)$, of the start of the sawtooth-free phase $\left(t_{2}\right)$ and of disruption $\left(t_{3}\right)$ are marked in accordance with figure 4 .

We note that while MARFE formation is a possibility at high density, appearance of MARFE was not clearly confirmed in these experiments.

\subsection{Effect of additional $E C H$}

EC heating has been used to investigate the possibility of density limit disruption prevention. A combination of X2 and X3 heating was used for this purpose. The cut-off density (local value) for the TCV X2 system is $n_{\mathrm{e}}^{\text {cut-off }}=4 \times 10^{19} \mathrm{~m}^{-3}$, and for the X3 system $n_{\mathrm{e}}^{\text {cut-off }}=1.1 \times 10^{20} \mathrm{~m}^{-3}$. Discharges with $I_{\mathrm{p}}=200 \mathrm{kA}$ have been investigated. Typical traces of plasma current and line averaged density are presented in figure 11. Traces of injected ECH power are also given in figure 11(c).

The heating power profiles and absorbed power fractions have been evaluated using the TORAY-GA ray-tracing code [14]. The absorbed power fraction for $\mathrm{X} 2$ appeared to be close to $100 \%$. For X3 the absorbed power fraction is found to be highest $(\sim 70 \%)$ in the presence of $\mathrm{X} 2$ heating due to the higher $T_{\mathrm{e}}$ (and could be even higher if a synergy effect were to occur [15]) and to decrease with density increase (figure 12), down to $3.5 \%$ at $\bar{n}_{\mathrm{e}} \approx 7 \times 10^{19} \mathrm{~m}^{-3}$. According to TORAY-GA calculations, this is accompanied by a displacement of the $\mathrm{X} 3$ heating region from localized on-axis to slightly off-axis, remaining however always inside the $q=1$ surface (figure 13).

The main effect of the X3 heating is obviously seen in the sawtooth dynamics (figure 14). The sawtooth-free phase is delayed, leading to an increase in the maximal achievable density. Table 2 contains a summary of the sawtooth behavior in high density discharges with and without sawteeth. It is seen that the application of X3 power inside the $q=1$ region leads to a significant delay in the sawtooth-free phase development. The plasma density corresponding to the start of the sawtooth-free phase increases up to $15 \%$. The subsequent disappearance of sawteeth and the eventual density limit disruption may be a result of the reduction in $\mathrm{X} 3$ absorption due to the further density increase. 

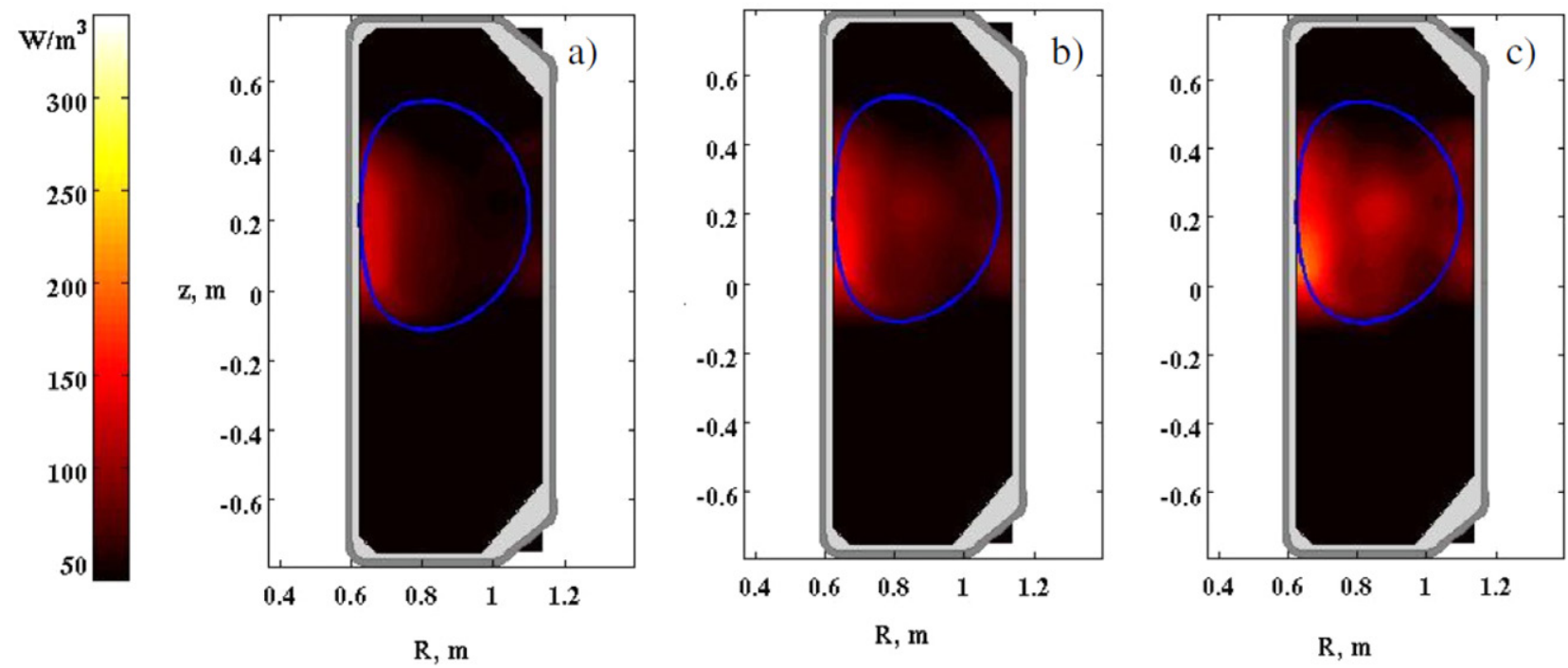

Figure 10. TCV shot 45181 -ohmic shot with a density limit disruption, $I_{\mathrm{p}}=200 \mathrm{kA}$ (similar to 45180 with the same timing). $2 \mathrm{D}$ reconstruction of bolometer data: $(a) t=1.1 \mathrm{~s}$ (phase with regular sawteeth), $(b) t=1.49 \mathrm{~s}$ (phase with modified sawtooth shape, before the start of sawtooth-free phase), $(c) t=1.65 \mathrm{~s}$ (sawtooth-free phase, close to disruption).

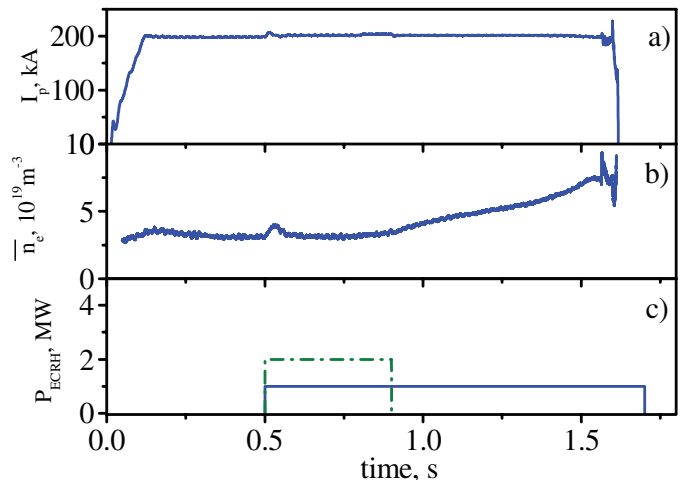

Figure 11. Typical scenario of high density experiments with application of ECH in TCV. Traces of plasma current $(a)$, line averaged plasma density measured along the central chord $(b)$ and injected ECH power $(c)$. The dashed-dotted trace on figure $11(c)$ is for the $\mathrm{X} 2$ power, the solid one for $\mathrm{X} 3$ power

\section{Modeling of the current profile evolution}

To analyze the effect of sawtooth disappearance and the role of EC heating, the current density profile dynamics were modeled using the ASTRA transport code [11]. The input quantities (temperature and density profiles, $Z_{\text {eff }}$ ) were derived as described in section 3. Kadomtsev's sawtooth model [16] was used for the profile modification due to sawtooth crashes. The two shots presented in figure 14, 45180 (pure ohmic heating) and 45184 (ohmic heating + ECRH), were modeled. Several sets of calculations were performed: (1) dynamic calculation following the experimental evolution of plasma parameters in the interval 1.4$1.65 \mathrm{~s}$ (for shot 45180) and (2) static modeling of discrete time instants using the experimental density and electron temperature distribution. The resulting $q(r)$ profiles are shown in figure 15 . Four time instants are presented for the shot 45180 - during the sawtoothing phase, at the beginning of the sawtooth free period and close to the disruption. The error bars on the $q(r)$ profiles depict the difference between the dynamic and static calculations. The radial distribution of $q(r)$ for shot 45184 is given at the time at which the measured value of the line averaged plasma density is close to $\left(\bar{n}_{\mathrm{e}}\right)_{\mathrm{SF}}$ for shot 45180 .

These simulations show that the density modification and the associated flattening of the temperature profile lead to a pronounced modification of the plasma current distribution. Based on the modeling results the q profiles in the sawtooth-free phase changes between the dashed blue and solid gray profiles in figure 15 , i.e. with a small $q=1$ radius and/or low shear near $q=1$ or no $q=1$ surface at all (as in the solid gray profile), consistent with sawtooth stabilization. These findings are consistent with the changes of $T_{\mathrm{e}}$ profiles discussed in [17], where first a change in $T_{\mathrm{e}}$ up to the mixing radius is observed, with a flattening of $T_{\mathrm{e}}$ and peaking of $n_{\mathrm{e}}$, and then a significant drop occurs in the whole $T_{\mathrm{e}}$ profile near the disruption density, due to changes near the edge. A more refined analysis of $q(r)$ profile would require taking into account modifications in the $Z_{\text {eff }}$ profile and applying a more complete sawtooth triggering model (for example, [18]).

Application of ECRH power inside the $q=1$ surface (see figure 13) clearly prevents $n_{\mathrm{e}}(r)$ peaking (the well-known density pump-out effect) and $T_{\mathrm{e}}(r)$ flattening (figure 16) and is expected to lead to restoration of a $q(r)$ profile with values well below 1 on axis. This is confirmed by ASTRA calculations made for the shot 45184 , as depicted by the dasheddotted line in figure 15 . This agrees well with the experimental observation that X3 heating maintains regular sawtooth oscillations at higher density.

\section{Confinement properties of high density ohmic plasmas}

The high-density discharges discussed in this paper were analyzed also with respect to their confinement properties. The following questions were addressed in particular: 

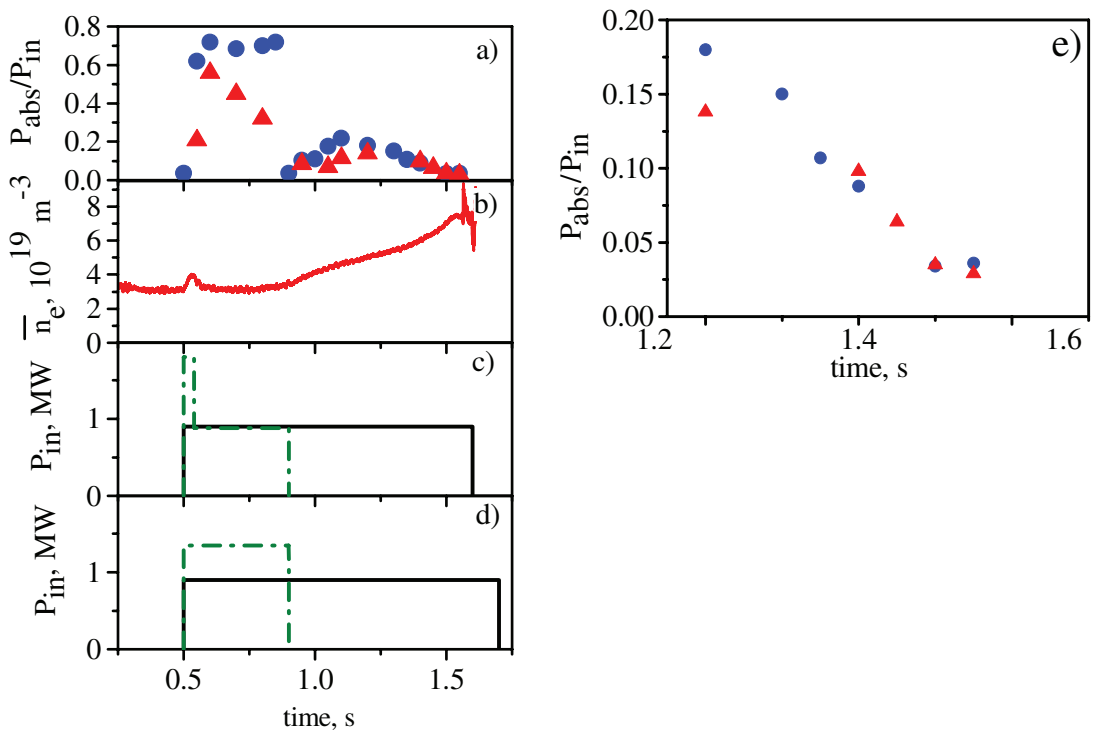

Figure 12. (a) Time evolution of the absorbed X3 power fraction in two similar discharges with high density: triangles-shot 45184 , circles-shot 45185; (b) line-averaged density behavior in shot 45184. Traces of injected ECRH power from X2 (dashed-dotted curves) and X3 (solid curves) systems for shots $45184(c)$ and $45185(d)$. Time evolution of absorbed power fraction in the high density phase and in the vicinity of the disruption (e): triangles—shot 45184, circles—shot 45185.

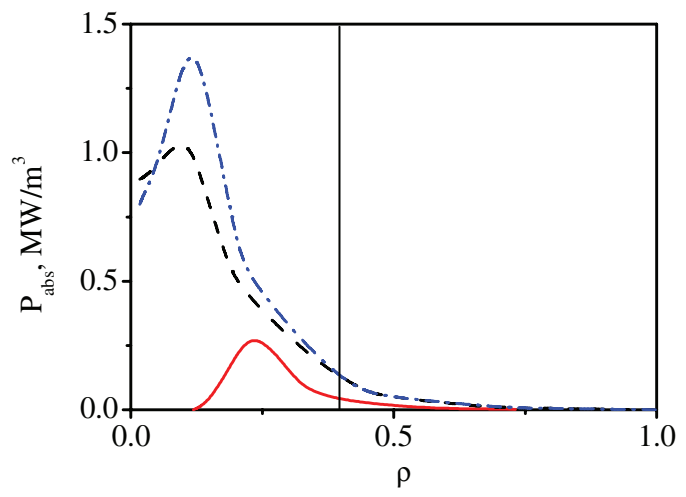

Figure 13. $\mathrm{X} 3$ absorbed power profiles evaluated from TORAY-GA calculations for the TCV shot presented in figure 10. Dashed curve $-t=1.1 \mathrm{~s}$, dashed-dotted curve $-t=1.2 \mathrm{~s}$, solid curve $-t=1.5 \mathrm{~s}$. $q=1$ position evaluated from equilibrium is marked by vertical solid line.

(a) do the confinement properties change with the density rise, and if so does the change occur at a particular threshold density?

(b) does confinement degrade in the immediate vicinity of the density limit?

(c) are there any differences between the behavior of particle and energy confinement?

The dependence of the global energy confinement time on line averaged density is presented in figure 17 for both moderate $(a)$ and low $(b)$ plasma current cases. The energy confinement time is derived from the plasma stored energy and the ohmic heating power:

$\tau_{E}=W_{\text {dia }} /\left(I_{\mathrm{p}} \cdot U_{\mathrm{L}}\right)$, where $W_{\mathrm{dia}}$ - stored energy from diamagnetic measurements, $U_{\mathrm{L}}$-loop voltage.

There are three typical confinement ranges in the case of higher plasma current:

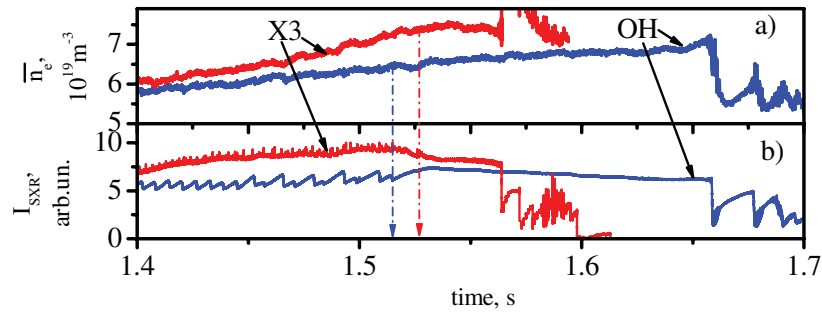

Figure 14. Comparison of plasma behavior in an ohmic shot (45180) and a shot with X3 heating (45184). Traces of line averaged plasma density $(a)$ and SXR emission measured along a central chord $(b)$. Starts of sawtooth-free phase for each shot are marked by dashed-dotted lines.

(a) linear ohmic confinement (LOC) at low densities up to $\bar{n}_{\mathrm{e}}=(4-4.5) \times 10^{19} \mathrm{~m}^{-3} \sim 0.4 n_{\mathrm{GW}}$;

(b) saturated ohmic confinement (SOC) at $\bar{n}_{\mathrm{e}} \simeq(6-6.3) \times 10^{19} \mathrm{~m}^{-3} \sim 0.6 n_{\mathrm{GW}}$ where the sawtoothfree phase starts;

(c) monotonic decrease of the confinement time after the start of the sawtooth-free phase.

In the case of low plasma current the initial steady-state density before the beginning of the ramp was higher than $0.4 n_{\mathrm{GW}}$ and no LOC phase is observed. A pronounced confinement time degradation is observed again after sawtooth stabilization.

Together with the tendencies in modification of energy confinement with the density growth, global changes in the density profile are also important giving some additional experimental background to test different theoretical approaches to explain the origin of anomalous transport in tokamaks. The temporal evolution of the density peaking is shown in figure 9 for a representative shot with moderate $q_{95}\left(q_{95}=3.7, I_{\mathrm{p}}=200 \mathrm{kA}\right)$. The density peaking factor $F(a / 2)$ as a function of line 
Table 2. Limit density and density corresponding to the start of the sawtooth-free phase for different shots with different heating

\begin{tabular}{llllll}
\hline Shot No & Heating type & $n_{\lim }\left(10^{19} \mathrm{~m}^{-3}\right)$ & $n_{\text {lim }} / n_{\mathrm{GW}}$ & $\left(\bar{n}_{\mathrm{e}}\right)_{\mathrm{SF}}\left(10^{19} \mathrm{~m}^{-3}\right)$ & $\left(\bar{n}_{\mathrm{e}}\right)_{\mathrm{SF}} / n_{\text {lim }}$ \\
\hline 45180 & $\mathrm{OH}$ & 7.0 & 0.62 & 6.5 & 0.9 \\
45181 & $\mathrm{OH}$ & 7.2 & 0.63 & 6.5 & 0.9 \\
45182 & $\mathrm{X} 3$ & 7.2 & 0.63 & 7.1 & 0.99 \\
45183 & $\mathrm{X} 3$ & 7.4 & 0.65 & 7.0 & 0.95 \\
45184 & $\mathrm{X} 2+\mathrm{X} 3$ & 7.45 & 0.65 & 7.45 & 1 \\
\hline
\end{tabular}

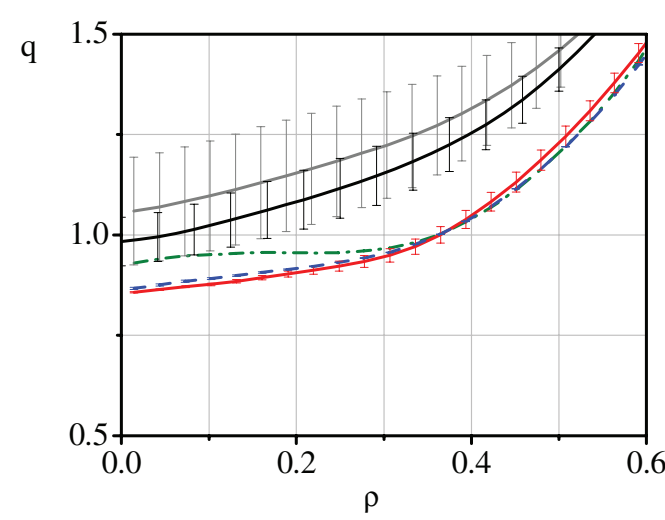

Figure 15. $q(r)$ profiles calculated by ASTRA code for shots presented in figure 14. Shot 45180: solid red curve: $t=t_{1}=1.45 \mathrm{~s}$, dashed blue curve: $t=1.57 \mathrm{~s}$, black solid curve: $t=1.60 \mathrm{~s}$, gray solid curve: $t=1.64 \mathrm{~s}$. TCV shot with X3 additional heating (45184), $t=1.5 \mathrm{~s}$ - green dashed-dotted curve.

averaged plasma density is presented in figure 18 for a broad data set with $q_{95}=3.7$ and $q_{95}=5\left(I_{\mathrm{p}}=150 \mathrm{kA}\right)$.

In the experiments discussed here a density increase is equivalent to a collisionality increase. The density peaking factor $F(a / 2)$ was evaluated from the FIR measurements in accordance with (2). For both data sets the density peaking factor does not decrease with the density increase. For moderate plasma current $\left(I_{\mathrm{p}}=200 \mathrm{kA}\right.$, figures $18(a)$ and $\left.(b)\right)$ the density peaking in fact increases with the increase of lineaveraged density up to $\bar{n}_{\mathrm{e}} \simeq 4 \times 10^{19} \mathrm{~m}^{-3} \sim 0.4 n_{\mathrm{GW}}$, close to the density range for the LOC-SOC transition; then it remains $\sim$ constant up to $\bar{n}_{\mathrm{e}} \simeq 6 \times 10^{19} \mathrm{~m}^{-3} \sim 0.6 n_{\mathrm{GW}}$, i.e. the start of the sawtooth-free phase. The density peaking abruptly increases at densities close to the transition to the SF phase.

In the case of lower plasma current (figure 18(c) the picture appears to be different since the investigated density range lies within the SOC regime. The variation of the density peaking at low density is less pronounced. However some tendencies can be noted. The density peaking starts to increase in the density range $\bar{n}_{\mathrm{e}} \sim(4-4.7) \times 10^{19} \mathrm{~m}^{-3} \sim(0.55-0.65) n_{\mathrm{GW}}$ for different shots, corresponding to the start of sawtooth suppression. At the density value $\bar{n}_{\mathrm{e}} \simeq 5 \times 10^{19} \mathrm{~m}^{-3} \simeq 0.7 n_{\mathrm{GW}}$ flattening of the density profile becomes pronounced, that is proposed to be a result of developed MHD activity leading to the internal crashes discussed in section 4.1.

\section{Conclusions and discussion}

The density limit process was investigated in TCV plasmas in ohmic and EC-heated discharges for different plasma
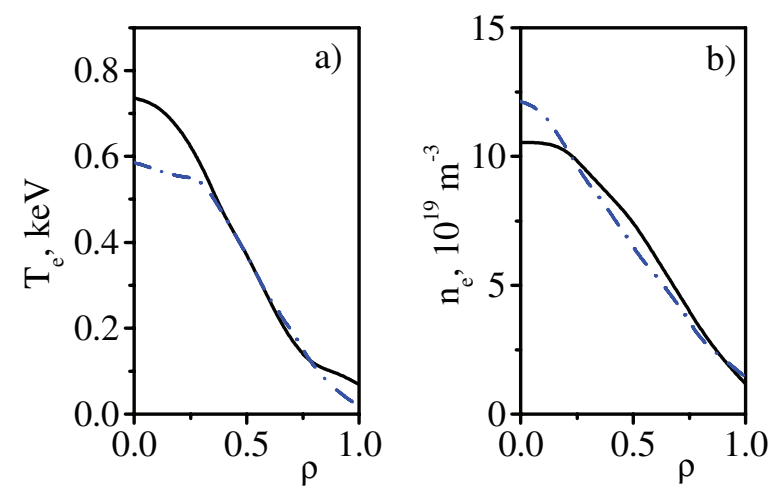

Figure 16. Electron temperature $(a)$ and density $(b)$ profiles measured by Thomson scattering. Dashed-dotted line: shot 45180: $t=1.60 \mathrm{~s}$, solid line: shot $45184, t=1.5 \mathrm{~s}$.

current levels. A density limit close to the Greenwald limit was reached at a high $q_{95}$ value of 7 , whereas at $q_{95} \sim 3.7$ the limit is lower $\left(0.6 n_{\mathrm{GW}}\right)$. The density limit appears to be caused by plasma current redistribution in the vicinity of the $q=1$ surface, with a temporary stabilization of sawteeth preceding the disruption. The density limit process in these TCV experiments developed as a giant sawtooth-like event terminating a long ( $100 \mathrm{~ms})$ sawtooth-free period. Accordingly, sawtooth destabilization by EC heating was shown to increase the density limit in this scenario. At low plasma current $\left(I_{\mathrm{p}} \leq 150 \mathrm{kA}\right.$, $q_{95} \geq 5$ in experiments discussed here) the density increase leads to a sawtooth-free phase accompanied by several minor disruptions.

The sequence of the events leading to the disruption can be described as follows:

(a) the density increase leads to a decrease in plasma temperature and core density peaking, resulting in turn in temperature profile flattening;

(b) the temperature profile flattening causes a flattening of the plasma current profile and a decrease of the magnetic shear near the $q=1$ surface, which changes the sawtooth behavior (increase of period, irregularity) until full sawtooth suppression occurs;

(c) the increase in sawtooth period and the eventual sawtooth disappearance leads to a further density increase in the core, to impurity accumulation and to an increase in core radiation losses; this causes further flattening of the plasma current profile with a decrease in magnetic shear near the $q=2$ surface followed by destabilization of a $m=2 / n=1$ mode;

(d) the growth of the $m=2 / n=1$ MHD island leads to the disruption. 

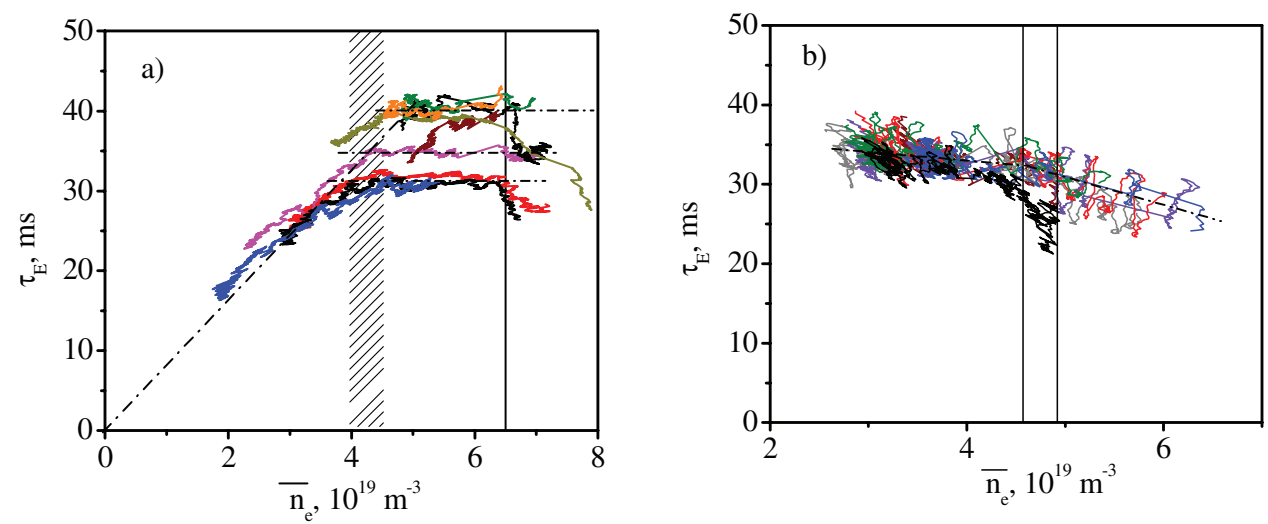

Figure 17. Dependence of the total energy confinement time on plasma density $(a) I_{\mathrm{p}}=200 \mathrm{kA}(b) I_{\mathrm{p}}=150 \mathrm{kA}$. Each curve corresponds to a different TCV shot. The dashed area in $(a)$ marks the transition from the linear ohmic confinement to the saturated ohmic confinement regime. The density at the start of the sawtooth-free phase is marked by the vertical solid line in $(a)$ and two solid lines in $(b)$ signifying a range of densities for the various shots in the set.
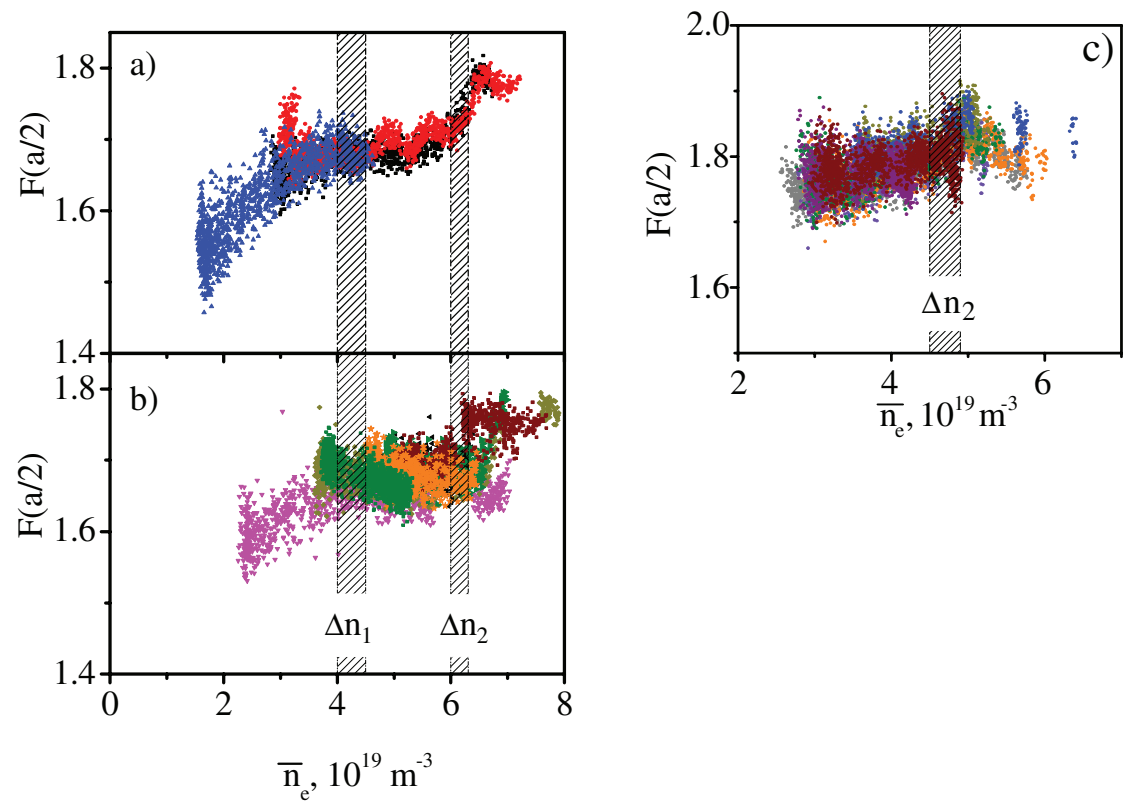

Figure 18. Dependence of the density peaking factor on plasma density $(a),(b) I_{\mathrm{p}}=200 \mathrm{kA}$ : for convenience the data are split into two plots for data taken in the 2011 (a) and 2013 (b) experimental campaigns, respectively; (c) $I_{\mathrm{p}}=150 \mathrm{kA}$. The $\Delta n_{1}$ range represents the density range of LOC-SOC transition, the $\Delta n_{2}$ range represents the start of sawtooth-free phase.

Central EC heating prevents temperature profile flattening and therefore current profile flattening, permitting an increase in the limit density value. This picture agrees well with the disruption model developed in [9]. In accordance with [9] prevention of the magnetic shear decrease by additional core heating and/or sawtooth destabilization should prevent the disruption. Note that this is also consistent with disruptions related to impurity accumulation in the center, which flatten the central temperature and which can be prevented by central heating.

The dependence of the confinement properties on the density increase has been investigated in ohmic plasmas. A transition from a LOC to a SOC regime has been observed at $\bar{n}_{\mathrm{e}} / n_{\mathrm{GW}} \sim 0.4$ at $I_{\mathrm{p}}=200 \mathrm{kA}\left(q_{95} \sim 3.7\right)$. The sawtooth stabilization with further density increase and the transition to the sawtooth-free phase were found to be accompanied by a decrease in the energy confinement time. This is consistent with recent results on the role of the modifications of the edge transport properties with increasing density at high density [17].

The density profile shape also changes with the density increase. The core density peaking factor does not decrease with the increase of the line-averaged density: on the contrary, it increases in the low density range (up to densities close to the LOC-SOC transition), abruptly increases at the beginning of sawtooth free phase and remains practically constant between of these two density ranges. This result is similar to that discussed in $[7,8]$.

Confinement modification and core density peaking with the density increase can be qualitatively explained based on the effect of drift turbulence on anomalous transport. In the experimental regimes discussed in this paper the TEM 
mode can be expected to dominate turbulence at low density. Density (=collisionality) increase leads to TEM suppression due to detrapping and to the transition to the ITG dominated regime. A quantitative estimation of the collisionality effect and theoretically based analysis of the balance between diffusive and non-diffusive particle flux in these experiments is outside the scope of this paper and will be the subject of future investigations.

\section{Acknowledgments}

First author (NK) would like to thank Dr A M Stefanovskii and Dr S V Mirnov for useful discussions.

This work was carried out in the framework of the 'Scientific and Technological Cooperation Programme Switzerland Russia, Utilization of Specific Infrastructure Projects'. This work was supported in part by the Swiss National Science Foundation. Russian participants of the work were also supported by the Rosatom (Contract No. H.4x.44.90.13.1101).

\section{References}

[1] ITER Physics Basis 1999 Nucl. Fusion 392137

[2] Greenwald M et al 1988 Nucl. Fusion 282199
[3] Alikaev V V et al 1990 Proc. 17th EPS Conf. on Plasma Physics and Controlled Fusion (Amsterdam, The Netherlands, 1990) vol 14B, Part III, p 1080

[4] Esipchuk Yu V et al 2003 Plasma Phys. Control. Fusion 45793

[5] Greenwald M 2002 Plasma Phys. Control. Fusion 44 R27

[6] Garbet X et al 2004 Plasma Phys. Control. Fusion 46 B557

[7] Kirneva N A et al 2008 Plasma Phys. Control. Fusion 50065004

[8] Wágner D et al 2012 Plasma Phys. Control. Fusion 54085018

[9] Mirnov S V 2001 Proc. 28th EPS Conf. on Control. Fusion and Plasma Physics (Funchal, Portugal, 18-22 June 2001) vol 25A (ECA) p 1473

[10] Gates D A and Delgado-Aparicio L 2012 Phys. Rev. Lett. 108165004

[11] Pereverzev G V and Yushmanov P N 2002 ASTRA-Automated System for TRansport Analysis IPP 5/98 February 2002

[12] Sauter O et al 1999 Phys. Plasmas 62834 Sauter O et al 2000 Phys. Plasmas 73122

[13] Canal G P et al 2012 Proc. 39th EPS Conf. and 16th Int. Congress on Plasma Physics (Stockholm, Sweden 2-6 July 2012) P5.076

[14] Matsuda K 1989 IEEE Trans. Plasma Sci. 176

[15] Alberti S et al 2000 Proc. 18th IAEA Fusion Energy Conf. (Sorrento, Italy, 2000) IAEA-CN-77/EXP4/04

[16] Kadomtsev B B 1976 Sov. J. Plasma Phys. 1389

[17] Sauter O et al 2014 Phys. Plasmas 21055906

[18] Porcelli F, Boucher D and Rosenbluth M 1996 Plasma Phys. Control. Fusion 38793 